\title{
TRIAZOLOPHTHALAZINE INCORPORATING PIPERAZINE DERIVATIVES: SYNTHESIS AND IN VITRO ANTICANCER EVALUATION STUDY
}

\author{
Abdallah Turky ${ }^{1}$, Ashraf H. Bayoumi ${ }^{1}$, Adel Ghiaty ${ }^{1}$, Hamada S. Abulkhair ${ }^{1,2 *}$ \\ ${ }^{1}$ Department of Pharmaceutical Organic Chemistry, Faculty of Pharmacy, Al-Azhar \\ University, Cairo, Egypt \\ ${ }^{2}$ Department of Pharmaceutical Chemistry, Faculty of Pharmacy, Horus University, \\ New Damietta, Egypt \\ *Corresponding author: hamadaorganic@azhar.edu.eg
}

\begin{abstract}
Cancer remains as one of the top leading causes of death worldwide in the last decade. In an attempt to develop a potent anticancer agent, herein we are reporting the synthesis of two novel series of piperazinyltriazolophthalazines as potential anticancer agents with potential inhibitory activity against PARP-1. All the newly synthesized compounds were

evaluated for their anti-proliferative activity against four human cancer cell lines namely; Hepatocellular carcinoma (HePG-2), Mammary gland breast cancer (MCF-7), Human prostate cancer (PC3) and Colorectal carcinoma (HCT-116)). The results of cytotoxicity evaluation showed that most of the synthesized compounds displayed moderate cytotoxic activity against the selected cell lines. Compound $\mathbf{2 3}$ showed the highest inhibitory effect followed by compound $\mathbf{2 4}$ against hepatocellular carcinoma (HePG2) with $\mathrm{IC}_{50}$ values of 15.05 and $17.23 \mu \mathrm{M}$ respectively. The same two compounds also showed moderate activity against colorectal carcinoma cell line (HCT116) with $\mathrm{IC}_{50}$ values of 21.93 and $24.06 \mu \mathrm{M}$ respectively.
\end{abstract}

Keywords: anticancer, piperazinyltriazolophthalazines, Hepatocellular carcinoma, Mammary gland breast cancer, Human prostate cancer and Colorectal carcinoma.

\section{Introduction}

Phthalazine derivatives are biologically important heterocycles and are known to possess variety of biological activities such as antitumor (Fedorov et al. 2014), anticonvulsant (Sun et al. 2011; Sun, Wei, et al. 2010), anti-inflammatory (Abd alla et al. 2010; Liu et al. 2016; Sun, Hu, et al. 2010), antidiabetic (Awadallah, El-Eraky, and Saleh 2012), antihypertensive (Olmo et al. 2006), muscle relaxant (Haack et al. 2005) and antimicrobial (Holló et al. 2014; Khalil, Berghot, and Gouda 2009; Salvi et al. 2006). Phthalazine scaffold is in the core structure of many commercially available drugs (Asif 2012) like, zopolrestat, azelastine, hydralazine and budralazine, the first is antidiabetic, the second is antihistaminic while the third and the last are vasodilating. A promising class of anticancer agents with micromolar range of $\mathrm{IC}_{50}$ 's is the 1,2,4triazoles (Lu et al. 2018). Triazolopthalazine rings presented in many anticancer agents 
with potent bromodomain inhibitory effects (Almahli et al. 2018; Boraei et al. 2019; Moustakim, Peter G. K. Clark, et al. 2017). Few years ago, compound 1 (L-45) was discovered as the first potent $[1,2,4]$ triazolo[3,4-a]phthalazine as anticancer with PCAF bromodomain inhibitory effect (Moustakim, Peter G. K. Clark, et al. 2017). Additionally, [1,2,4]triazolo[3,4-a]phthalazine is the pharmacophore ring system of certain anticancer agents (El-Helby et al. 2018; Moustakim, Peter G.K. Clark, et al. 2017; Xue et al. 2014). In the last decade, many synthetic [1,2,4]triazolo[3,4a]phthalazines (Figure 1) have been reported to exhibit potent anticancer activities against hepatocellular carcinoma with $\mathrm{IC}_{50}$ values range of 1.60-6.04 (El-Helby et al. 2017, 2018; Xue et al. 2014).<smiles>Cc1nnc2c3ccccc3c(N[C@H](C)[C@H](c3ccccc3)N(C)C)nn12</smiles>

$L-45,1$

$\mathrm{IC}_{50}: 1.60 \mu \mathrm{M}$<smiles>O=C(NNc1nn2cnnc2c2ccccc12)Nc1ccccc1</smiles>

2

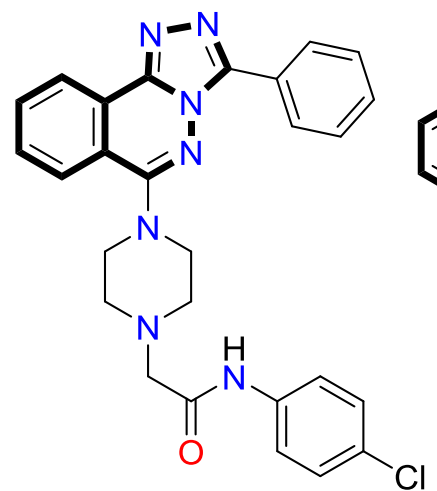

3<smiles>CC(=O)Nc1ccccc1F</smiles>

$\mathrm{IC}_{50}: 4.50 \mu \mathrm{M}$

Figure 1: Structures of selected triazolophthalazines with potent anticancer activity against hepatocellular carcinoma.

On the other hand, piperazines are important class of compounds with diverse biological activities, particularly as anticancer (Chetan et al. 2010; Gurdal et al. 2015). Piperazine derivatives $5 \& \mathbf{6}$ (Figure 2) showed potent cytotoxic activity against NCIH460, HCT116 and U251 cell lines. Considering the above-mentioned facts and as an attempt to develop a new molecule with potent anticancer activity, molecular hybridization between triazolophthalazine scaffold and piperazine moiety was carried out. Here, inspired by the versatility of the[1,2,4]triazolo[3,4-a]phthalazine pharmacophoric ring skeleton of $\boldsymbol{L} \mathbf{- 4 5}$ and the piperazine moiety mentioned above, nine novel compounds were synthesized to evaluate their anticancer activities. Different substitution patterns were introduced to both the C-3 of triazolophthalazine scaffold and the terminal pierazine ring to investigate the effect on cytotoxicity of the synthesized compounds. All the synthesized compounds were evaluated for their in vitro anticancer activity against four cancer cell lines namely; hepatocellular carcinoma (HePG-2), mammary gland breast cancer (MCF-7), human prostate cancer (PC3) and colorectal carcinoma (HCT-116). 
<smiles>O=C(NO)N1CCN(C(=S)N/N=C/c2c3ccccc3cc3ccccc23)CC1</smiles>

5<smiles>O=C(NO)N1CCN(C(=S)N/N=C/c2cccc(O)c2)CC1</smiles>

6

Figure 2: Structures of some reported anticancer agents incorporating piperazine ring.

\section{Results and discussion}

Chemistry: The general route for the synthesis of starting triazolophthalazines is illustrated in Scheme 1. 4-Methylbenzoic acid converted into the ethyl ester by the action of ethanol in the presence of catalytic amount of sulfuric acid. The resulting ester allowed to react with hydrazine hydrate in refluxing ethanol to get 4methylbenzohydrazide (9). In a parallel pathway, phthalic anhydride reacted with hydrazine hydrate in acetic acid to yield 2,3-dihydrophthalazine-1,4-dione (11). The latter reacted under reflux with phosphorus oxychloride to yield 1,4-dichlorophthalazine (12). Treating compound with 12 hydrazine hydrate at room temperature in ethanol to produce 1-chloro-4-hydrazinylphthalazine (13) (Abou-Seri et al. 2016). Reaction of the hydrazinyl derivative $\mathbf{1 3}$ with trifluoroacetic acid followed by chlorination with phosphorus oxychloride yielded 6-chloro-3-(trifluoromethyl)-[1,2,4]triazolo[3,4a]phthalazine (14a). Alternatively, treating the dichlorophthalazine derivative 12 with 4-methylbenzohydrazide in refluxing ethanol produced the triazolophthalazine derivatives $\mathbf{1 4 b}$. 
<smiles>CCOC(=O)c1ccc(C)cc1</smiles><smiles>O=C1OC(=O)c2ccccc21</smiles>

10<smiles>O=c1[nH][nH]c(=O)c2ccccc12</smiles>

11<smiles>Cc1ccc2c(Cl)nnc(Cl)c2c1</smiles>

12<smiles>Cc1ccc(-c2nnc3c4ccc(C)cc4c(Cl)nn23)cc1</smiles>

14b

Reagents and conditions:<smiles>NNc1nnc(Cl)c2ccccc12</smiles>

13<smiles>C[GeH3]</smiles><smiles>FC(F)(F)c1nnc2c3ccccc3c(Cl)nn12</smiles>

$14 a$

a) $\mathrm{C}_{2} \mathrm{H}_{5} \mathrm{OH}$ (absolute) $/ \mathrm{H}_{2} \mathrm{SO}_{4}, 100{ }^{\circ} \mathrm{C} 77 \%$. b) $\mathrm{H}_{2} \mathrm{~N}-\mathrm{NH}_{2} \cdot \mathrm{H}_{2} \mathrm{O}, \mathrm{C}_{2} \mathrm{H}_{5} \mathrm{OH}, 100{ }^{\circ} \mathrm{C}, 72 \%$.

c) $\mathrm{H}_{2} \mathrm{~N}-\mathrm{NH}_{2} \cdot \mathrm{H}_{2} \mathrm{O}, \mathrm{CH}_{3} \mathrm{COOH}, 120^{\circ} \mathrm{C}, 85 \%$; d) $\mathrm{POCl}_{3}, 110{ }^{\circ} \mathrm{C}, 5 \mathrm{~h}, 90 \%$.

e) $4-\mathrm{CH}_{3} \mathrm{C}_{6} \mathrm{H}_{4} \mathrm{CONHNH}_{2}(9)$, dioxane, $140{ }^{\circ} \mathrm{C}, 6 \mathrm{~h}, 80 \%$

f) $\mathrm{H}_{2} \mathrm{~N}-\mathrm{NH}_{2} \cdot \mathrm{H}_{2} \mathrm{O}, \mathrm{C}_{2} \mathrm{H}_{5} \mathrm{OH}, \mathrm{rt}, 3 \mathrm{~h}, 70 \%$; g) $\mathrm{CF}_{3} \mathrm{COOH}, 140 \circ 10 \mathrm{~h}, 85 \%$.

Scheme 1: Synthetic route for preparation of starting triazolophthalazines

Reaction of the triazolophthalazine derivatives $\mathbf{1 4 a , b}$ with piperazine in ethanol according to reported procedures (Tarzia et al. 1988) afforded the piperazinyl derivatives 15a,b (Scheme 2). Our final target compounds were readily obtained in good yields and reasonable purities by treating the latter with the appropriate acid chloride. 
<smiles>[R]c1nnc2c3ccccc3c(Cl)nn12</smiles>

$14 a, b$<smiles>[R]c1nnc2c3ccccc3c(N3CCN([Y5])CC3)nn12</smiles>

, 6-10 h, $80 \%$.<smiles>[R]C(=O)N1CCN(c2nn3c([R])nnc3c3ccccc23)CC1</smiles>

$16-24$

b) $\mathrm{RCOCl}, \mathrm{DMF}, 80{ }^{\circ} \mathrm{C}, 4 \mathrm{~h}, 66-80 \%$.

Scheme 2: Synthetic route for preparation of target piperazinyl triazolophthalazines

Anticancer evaluation: Anticancer activities of the synthesized compounds were assessed on four cancer cell lines namely; hepatocellular carcinoma (HePG-2), mammary gland breast cancer (MCF-7), human prostate cancer (PC3) and colorectal carcinoma (HCT-116) using MTT assay (Mosmann 1983). Doxorubicin was used as a reference anticancer agent. The results of preliminary anticancer evaluation are shown in Table 1. The results of cytotoxicity evaluation showed that the majority of the synthesized compounds displayed moderate cytotoxic activities against the selected cell lines. Compound $\mathbf{2 3}$ showed the highest inhibitory effect followed by compound $\mathbf{2 4}$ against hepatocellular carcinoma (HePG2) with $\mathrm{IC}_{50}$ values of 15.05 and $17.23 \mu \mathrm{M}$ respectively. The same two compounds also showed moderate activity against colorectal carcinoma cell line (HCT-116) with $\mathrm{IC}_{50}$ values of 21.93 and $24.06 \mu \mathrm{M}$ respectively. Compounds with trifluoromethy group attached to C-3 of the triazolophthalazine system (16-19) displayed relatively lower inhibitory potencies than all other compounds. 
Table 1: In vitro anticancer activity of the new piperazinyl triazolophthalazines

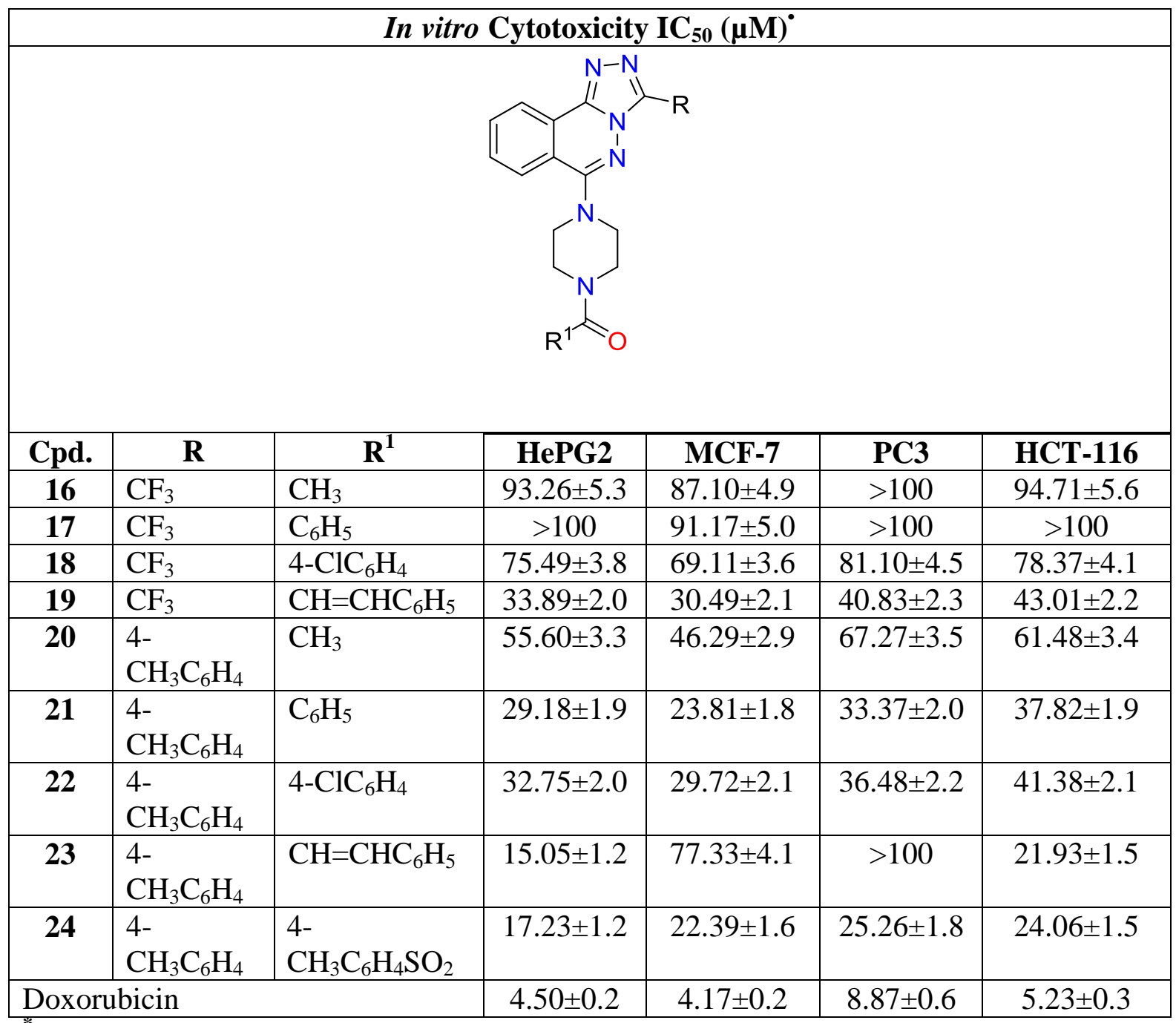

${ }^{*} \mathbf{I C}_{\mathbf{5 0}}(\boldsymbol{\mu M}): 1-10$ (very strong). $11-20$ (strong). $21-50$ (moderate). $51-100$ (weak) and above 100 (non-cytotoxic). 


\section{Experimental}

\section{General:}

All melting points were determined using capillary tubes with a Stuart SMP30 apparatus and are uncorrected. ${ }^{1} \mathrm{H}$ NMR spectra were recorded on Bruker-400-MHz spectrophotometer using DMSO- $\mathrm{d}_{6}$ as a solvent and TMS as internal reference. ${ }^{13} \mathrm{C}$ NMR spectra were recorded on Bruker-100-MHz spectrophotometer using DMSO- $\mathrm{d}_{6}$ as a solvent and TMS as internal reference. Chemical shifts were recorded in $\delta \mathrm{ppm}$ downfield the TMS signal. Mass spectra were recorded on Hewlett Packard 5988 spectrometer. Elemental analyses were performed on $\mathrm{CHN}$ analyzer. All spectral measurements have been performed at the Regional Center for Mycology \& Biotechnology, Al-Azhar University, Cairo, Egypt. Anticancer evaluation was carried out at the Holding Company for Biological Products and Vaccines, Egypt (Vacsera).

General procedure for preparation of (4-(3-substituted-[1,2,4]triazolo[3,4a]phthalazin-6-yl)piperazin-1-yl)(acyl)methanones (16-24):

Compounds $15(0.001 \mathrm{~mol})$ was dissolved in dimethyl formamide $(20 \mathrm{ml})$ and acetyl chloride $(0.01 \mathrm{~mol})$ or aryl chloride $(0.008 \mathrm{~mol})$ was added and the mixture was refluxed at $80^{\circ} \mathrm{C}$ for $3 \mathrm{~h}$. After the end of the reaction (monitored by TLC), the mixture was allowed to cool and poured on crushed ice. On standing, the precipitate was formed and collected by filtration. This solid was recrystallized from ethanol to afford the corresponding compounds.

\section{1-(4-(3-(Trifluoromethyl)-[1,2,4]triazolo[3,4-a]phthalazin-6-yl)piperazin-1- yl)ethan-1-one (16):}

White solid; Yield: 76\%; m.p.255-257 ${ }^{\circ} \mathrm{C}$. IR ( $\left.\mathrm{KBr}\right) \mathrm{cm}^{-1}: 3016$ (CH aromatic), 2974 ( $\mathrm{CH}$ aliphatic), $1643(\mathrm{C}=\mathrm{O}), 1589\left(\mathrm{C}=\mathrm{C}\right.$ aromatic). ${ }^{1} \mathrm{H}$ NMR (DMSO-d $\left.{ }_{6}\right) \delta \mathrm{ppm}$ : 8.54 (d, $1 \mathrm{H}, J=7.8), 8.21(\mathrm{~d}, 1 \mathrm{H}, J=8.1), 8.06$ (t, $1 \mathrm{H}, J=7.5), 7.97$ (t, $1 \mathrm{H}, J=7.6)$, $3.74(\mathrm{~s}, 4 \mathrm{H}), 3.74(\mathrm{~s}, 2 \mathrm{H}), 3.42(\mathrm{~s}, 2 \mathrm{H}), 2.08(\mathrm{~s}, 3 \mathrm{H}) . \mathrm{MS}(\mathrm{m} / \mathrm{z}): 364\left(\mathrm{C}_{16} \mathrm{H}_{15} \mathrm{~F}_{3} \mathrm{~N}_{6} \mathrm{O}\right.$, $\left.18.78 \%, \mathrm{M}^{+}\right) 295\left(\mathrm{C}_{15} \mathrm{H}_{15} \mathrm{~N}_{6} \mathrm{O}, 21.33 \%\right), 255\left(\mathrm{C}_{13} \mathrm{H}_{15} \mathrm{~N}_{6}, 16 \%\right), 127\left(\mathrm{C}_{6} \mathrm{H}_{11} \mathrm{~N}_{2} \mathrm{O}, 100 \%\right)$, $71\left(\mathrm{C}_{4} \mathrm{H}_{9} \mathrm{~N}, 24.3 \%\right) .{ }^{13} \mathrm{C}$ NMR (DMSO-d 6$) \delta \mathrm{ppm}: 169.10,158.80,144.66,139.26$, 138.86, 134.22, 132.15, 127.40, 123.83, 123.48, 120.40, 120.23, 117.55, 51.42, 51.01, 45.47, 40.77 and 21.62. Anal. Calc. for: $\left(\mathrm{C}_{16} \mathrm{H}_{15} \mathrm{~F}_{3} \mathrm{~N}_{6} \mathrm{O}\right)(\mathrm{M} . \mathrm{W} .=364): \mathrm{C}, 52.75 ; \mathrm{H}$, 4.15; F, 15.64; N, 23.07\%; Found: C, 53.02; H, 54.41; N, $22.89 \%$.

\section{Phenyl(4-(3-(trifluoromethyl)-[1,2,4]triazolo[3,4-a]phthalazin-6-yl)piperazin-1- yl)methanone (17):}

White. Yield: $67 \%$; m.p. $263-265^{\circ} \mathrm{C}$. IR (KBr) cm ${ }^{-1}: 3028$ (CH aromatic), 2997 ( $\mathrm{CH}$ aliphatic), $1624(\mathrm{C}=\mathrm{O}), 1589\left(\mathrm{C}=\mathrm{C}\right.$ aromatic). ${ }_{1} \mathrm{H}$ NMR (DMSO-d $\left.{ }_{6}\right) \delta \mathrm{ppm}: 8.59$ $(\mathrm{d}, 1 \mathrm{H}, J=7.76), 8.25(\mathrm{~d}, 1 \mathrm{H}, J=8.08), 8.08(\mathrm{t}, 1 \mathrm{H}, J=7.5), 7.99(\mathrm{t}, 1 \mathrm{H}, J=7.6), 7.49$ $(\mathrm{m}, 5 \mathrm{H}), 3.92(\mathrm{~s}, 2 \mathrm{H}), 3.65(\mathrm{~s}, 2 \mathrm{H}), 3.46(\mathrm{~s}, 4 \mathrm{H}) . \mathrm{MS}(\mathrm{m} / \mathrm{z}): 426\left(\mathrm{C}_{21} \mathrm{H}_{17} \mathrm{~F}_{3} \mathrm{~N}_{6} \mathrm{O}, 100 \%\right.$, $\left.\mathrm{M}^{+}\right) 321\left(\mathrm{C}_{14} \mathrm{H}_{12} \mathrm{~F}_{3} \mathrm{~N}_{6}, 4.74 \%\right), 292\left(\mathrm{C}_{13} \mathrm{H}_{9} \mathrm{~F}_{3} \mathrm{~N}_{5}, 2.74 \%\right), 104\left(\mathrm{C}_{7} \mathrm{H}_{6} \mathrm{~N}, 1.42 \%\right), 77$ $\left(\mathrm{C}_{6} \mathrm{H}_{5}, 26.84 \%\right),{ }^{13} \mathrm{C}$ NMR (DMSO-d $\left.{ }_{6}\right) \delta \mathrm{ppm}: 169.89,158.83,144.82,139.33,138.94$, $136.04,134.39$, 132.29, 130.15, 128.95, 127.5, 123.96, 123.58, 120.5, 117.56, 51.14 
and 40.45. Anal. Calc. for: $\left(\mathrm{C}_{21} \mathrm{H}_{17} \mathrm{~F}_{3} \mathrm{~N}_{6} \mathrm{O}\right)$ (M.W. = 426): C, 59.15; H, 4.02; F, 13.37; N, $19.71 \%$; Found: C, 59.38; H, 4.23; N, $19.87 \%$.

\section{(4-Chlorophenyl)(4-(3-(trifluoromethyl)-[1,2,4]triazolo[3,4-a]phthalazin-6- yl)piperazin-1-yl)methanone (18):}

White solid; Yield: $77 \%$; m.p.267-269 ${ }^{\circ} \mathrm{C}$. IR ( $\left.\mathrm{KBr}\right) \mathrm{cm}^{-1}: 3032$ (CH aromatic), 2997 (CH aliphatic), $1624(\mathrm{C}=\mathrm{O}), 1593\left(\mathrm{C}=\mathrm{C}\right.$ aromatic). ${ }^{1} \mathrm{H}$ NMR (DMSO-d 6 ) $\delta$ ppm: 8.59 (d, 1H, $J=7.4), 8.24$ (d, 1H, $J=8.1), 8.09$ (t, $1 \mathrm{H}, J=7.3), 7.99$ (t, $1 \mathrm{H}, J=8.2$ ), 7.56-7.51 (m, 4H), $3.92(\mathrm{~s}, 2 \mathrm{H}), 3.64(\mathrm{~s}, 2 \mathrm{H}), 3.46(\mathrm{~s}, 4 \mathrm{H})$. MS $(\mathrm{m} / \mathrm{z}): 462$ $\left(\mathrm{C}_{21} \mathrm{H}_{16} \mathrm{ClF}_{3} \mathrm{~N}_{6} \mathrm{O}, 31.81 \%, \mathrm{M}^{+}+2\right) 460\left(\mathrm{C}_{21} \mathrm{H}_{16} \mathrm{ClF}_{3} \mathrm{~N}_{6} \mathrm{O}, 100 \%, \mathrm{M}^{+}\right) 321\left(\mathrm{C}_{14} \mathrm{H}_{12} \mathrm{~F}_{3} \mathrm{~N}_{6}\right.$, $1.69 \%), 279\left(\mathrm{C}_{12} \mathrm{H}_{8} \mathrm{~F}_{3} \mathrm{~N}_{5}, 1.31 \%\right), 139\left(\mathrm{C}_{7} \mathrm{H}_{4} \mathrm{ClO}, 1.8 \%\right),{ }^{13} \mathrm{C}$ NMR (DMSO-d6) $\delta \mathrm{ppm:}$ $168.81,158.84,144.87,139.35,138.95,134.86,134.82,134.43,132.31,129.58$, 129.02, 127.55, 124.01, 123.66, 120.56, 120.26, 117.58, 51.09 and 40.51. Anal. Calc. for: $\left(\mathrm{C}_{21} \mathrm{H}_{16} \mathrm{ClF}_{3} \mathrm{~N}_{6} \mathrm{O}\right)(\mathrm{M} . \mathrm{W} .=460)$ : C, 54.73; H, 3.5; Cl, 7.69; F, 12.37; N, 18.24\%; Found: C, 54.91; H, 3.67; N, 18.07\%.

\section{3-Phenyl-1-(4-(3-(trifluoromethyl)-[1,2,4] triazolo[3,4-a]phthalazin-6-yl)piperazin- 1-yl)prop-2-en-1-one (19):}

White solid; Yield: 79\%; m.p.261-263․ $\mathrm{C}$. IR ( $\mathrm{KBr}) \mathrm{cm}^{-1}$ : $3001(\mathrm{CH}$ aromatic), 2893 (CH aliphatic), $1647(\mathrm{C}=\mathrm{O}), 1608\left(\mathrm{C}=\mathrm{C}\right.$ aromatic). ${ }^{1} \mathrm{H}$ NMR (DMSO-d $\left.{ }_{6}\right) \delta \mathrm{ppm}$ : $8.6(\mathrm{~d}, 1 \mathrm{H}, J=7.8), 8.27(\mathrm{~d}, 1 \mathrm{H}, J=8.1), 8.09(\mathrm{t}, 1 \mathrm{H}, J=7.1), 8(\mathrm{t}, 1 \mathrm{H}, J=7.4), 7.74$ $(\mathrm{d}, 2 \mathrm{H}, J=6.7), 7.55(\mathrm{~d}, 1 \mathrm{H}, \mathrm{J}=15.4), 7.44-7.34(\mathrm{~m}, 4 \mathrm{H}), 4.03(\mathrm{~s}, 2 \mathrm{H}), 3.89(\mathrm{~s}, 2 \mathrm{H})$, 3.47 ( s, 4H). MS (m/z): $452\left(\mathrm{C}_{23} \mathrm{H}_{19} \mathrm{~F}_{3} \mathrm{~N}_{6} \mathrm{O}, 100 \%, \mathrm{M}^{+}\right) 375\left(\mathrm{C}_{17} \mathrm{H}_{14} \mathrm{~F}_{3} \mathrm{~N}_{6} \mathrm{O}, 1.96 \%\right), 280$ $\left(\mathrm{C}_{12} \mathrm{H}_{9} \mathrm{~F}_{3} \mathrm{~N}_{5}, 2.63 \%\right), 131\left(\mathrm{C}_{8} \mathrm{H}_{7} \mathrm{~N}_{2}, 23.52 \%\right), 77\left(\mathrm{C}_{6} \mathrm{H}_{5}, 36.23 \%\right) .{ }^{13} \mathrm{C}$ NMR (DMSO-d $\left.\mathrm{d}_{6}\right)$ $\delta$ ppm: 165.24, 158.84, 144.83, 142.29, 139.33, 138.93, 135.52, 134.37, 132.27, 130.05, 129.21, 128.48, 127.54, 123.99, 123.65, 120.56, 120.28, 118.51, 117.6, 51.6 and 41.9. Anal. Calc. for: $\left(\mathrm{C}_{23} \mathrm{H}_{19} \mathrm{~F}_{3} \mathrm{~N}_{6} \mathrm{O}\right)(\mathrm{M} . \mathrm{W} .=452)$ : C, 61.06; H, 4.23; F, 12.6; N, 18.58\%; Found: C, 60.89; H, 4.42; N, 18.7\%.

\section{1-(4-(3-(p-Tolyl)-[1,2,4]triazolo[3,4-a]phthalazin-6-yl)piperazin-1-yl)ethan-1-one (20):}

White solid; Yield: $69 \%$; m.p. $259-261^{\circ} \mathrm{C}$. IR (KBr) cm ${ }^{-1}$ : 3024 (CH aromatic), 2951 (CH aliphatic), $1643(\mathrm{C}=\mathrm{O}), 1589\left(\mathrm{C}=\mathrm{C}\right.$ aromatic). ${ }^{1} \mathrm{H}$ NMR (DMSO-d $\left.{ }_{6}\right) \delta$ ppm: 8.53 (d, 1H, $J=7.7$ ), 8.27 ( d, 2H, $J=7.8$ ), 8.19 ( d, 1H, $J=8.0), 8.01$ ( t,1H, $J=7.4$ ), $7.9(\mathrm{t}, 1 \mathrm{H}, J=7.5), 7.41(\mathrm{~d}, 2 \mathrm{H}, J=7.7), 3.76(\mathrm{~s}, 4 \mathrm{H}), 3.41(\mathrm{~s}, 4 \mathrm{H}), 2.4\left(\mathrm{~s}, 3 \mathrm{H}, \mathrm{CH}_{3}\right)$, $2.09\left(\mathrm{~s}, 3 \mathrm{H}, \mathrm{COCH}_{3}\right)$. MS $(\mathrm{m} / \mathrm{z}): 386\left(\mathrm{C}_{22} \mathrm{H}_{22} \mathrm{~N}_{6} \mathrm{O}, 43.57 \%, \mathrm{M}^{+}\right) 330\left(\mathrm{C}_{20} \mathrm{H}_{20} \mathrm{~N}_{5}\right.$, 54.39\%), $261\left(\mathrm{C}_{16} \mathrm{H}_{13} \mathrm{~N}_{4}, 26.63 \%\right), 214\left(\mathrm{C}_{12} \mathrm{H}_{14} \mathrm{~N}_{4}, 100 \%\right), 124\left(\mathrm{C}_{6} \mathrm{H}_{10} \mathrm{~N}_{3}, 15.89 \%\right) .{ }^{13} \mathrm{C}$ NMR (DMSO-d $\left.{ }_{6}\right) \delta$ ppm: 169.06, 157.66, 148.1, 143.18, 140.05, 133.97, 131.15, 129.78, 127.58, 127.19, 124.63, 124.12, 123.49, 119.8, 51.35, 45.74, 21.69 and 21.48. Anal. Calc. for: $\left(\mathrm{C}_{22} \mathrm{H}_{22} \mathrm{~N}_{6} \mathrm{O}\right)(\mathrm{M} . \mathrm{W} .=386): \mathrm{C}, 68.38 ; \mathrm{H}, 5.74 ; \mathrm{N}, 21.75 \%$; Found: $\mathrm{C}$, $68.59 ; \mathrm{H}, 5.81 ; \mathrm{N}, 21.98 \%$. 


\section{Phenyl(4-(3-(p-tolyl)-[1,2,4]triazolo[3,4-a]phthalazin-6-yl)piperazin-1- yl)methanone (21):}

White solid; Yield: 67\%; m.p.261-263 ${ }^{\circ} \mathrm{C}$. IR ( $\left.\mathrm{KBr}\right) \mathrm{cm}^{-1}$ : $3028(\mathrm{CH}$ aromatic), $2993\left(\mathrm{CH}\right.$ aliphatic), $1635(\mathrm{C}=\mathrm{O}), 1589\left(\mathrm{C}=\mathrm{C}\right.$ aromatic). ${ }^{1} \mathrm{H}$ NMR (DMSO-d $\left.{ }_{6}\right) \delta \mathrm{ppm}$ : 8.55 (d, 1H, $J=7.9$ ), 8.27 ( d, 2H, $J=8.04$ ), 8.2 ( d, $1 \mathrm{H}, J=8.1$ ), 8.02 ( t,1H, $J=7.6$ ), 7.9 (t, $1 \mathrm{H}, J=7.5), 7.49(\mathrm{~m}, 5 \mathrm{H}), 7.42(\mathrm{~d}, 2 \mathrm{H}, J=7.9), 3.96(\mathrm{~s}, 2 \mathrm{H}), 3.69(\mathrm{~s}, 2 \mathrm{H}), 3.44($ $\mathrm{s}, 4 \mathrm{H}), 2.41$ ( s, 3H). MS (m/z): $448\left(\mathrm{C}_{27} \mathrm{H}_{24} \mathrm{~N}_{6} \mathrm{O}, 7.06 \%, \mathrm{M}^{+}\right) 301\left(\mathrm{C}_{18} \mathrm{H}_{15} \mathrm{~N}_{5}, 12.57 \%\right)$, $259\left(\mathrm{C}_{16} \mathrm{H}_{11} \mathrm{~N}_{4}, 6.33 \%\right), 105\left(\mathrm{C}_{7} \mathrm{H}_{5} \mathrm{O}, 100 \%\right) .{ }^{13} \mathrm{C}$ NMR (DMSO-d $\left.{ }_{6}\right) \delta \mathrm{ppm}: 169.79$, $157.61,148.16,143.21,140.14,136.11,134.02,131.22,130.16,129.83,128.98$, 127.63, 127.51, 127.23, 124.58, 124.03, 123.51, 119.77, 51.27, 40.45 and 21.47. Anal. Calc. for: $\left(\mathrm{C}_{27} \mathrm{H}_{24} \mathrm{~N}_{6} \mathrm{O}\right)(\mathrm{M} . \mathrm{W} .=448)$ : C, 72.3; H, 5.39; N, 18.74\%; Found: C, 72.52; H, $5.61 ; \mathrm{N}, 18.92 \%$.

(4-Chlorophenyl)(4-(3-(p-tolyl)-[1,2,4]triazolo[3,4-a]phthalazin-6-yl)piperazin-1-

yl)methanone (22): White solid; Yield: $74 \%$; m.p.263-265 ${ }^{\circ} \mathrm{C}$. IR (KBr) $\mathrm{cm}^{-1}: 3082$ (CH aromatic), 2993 (CH aliphatic), $1631(\mathrm{C}=\mathrm{O}), 1593\left(\mathrm{C}=\mathrm{C}\right.$ aromatic). ${ }^{1} \mathrm{H}$ NMR $\left(\mathrm{DMSO}_{-} \mathrm{d}_{6}\right) \delta \mathrm{ppm}: 8.52(\mathrm{~d}, 1 \mathrm{H}, J=7.6), 8.25(\mathrm{~d}, 2 \mathrm{H}, J=7.6), 8.17(\mathrm{~d}, 1 \mathrm{H}, J=7.9), 8$ $(\mathrm{t}, 1 \mathrm{H}, J=7.4), 7.88(\mathrm{t}, 1 \mathrm{H}, J=7.2), 7.54(\mathrm{~m}, 4 \mathrm{H}), 7.39$ ( d, 2H, $J=7.5), 3.94(\mathrm{~s}, 2 \mathrm{H})$, 3.66 (s, 2H), 3.43 ( s, 4H), 2.39 ( s, 3H). MS (m/z): $484\left(\mathrm{C}_{27} \mathrm{H}_{23} \mathrm{ClN}_{6} \mathrm{O}, 33.56 \%, \mathrm{M}^{+}+2\right)$ $482\left(\mathrm{C}_{27} \mathrm{H}_{23} \mathrm{ClN}_{6} \mathrm{O}, 64.67 \%, \mathrm{M}^{+}\right) 343\left(\mathrm{C}_{20} \mathrm{H}_{19} \mathrm{~N}_{6}, 12.31 \%\right), 259\left(\mathrm{C}_{16} \mathrm{H}_{11} \mathrm{~N}_{4}, 58.37 \%\right)$, $144\left(\mathrm{C}_{8} \mathrm{H}_{6} \mathrm{~N}_{3}, 100 \%\right) .{ }^{13} \mathrm{C}$ NMR (DMSO-d 6$) \delta$ ppm: 148.05, 134.18, 130.71, 129.15, 127.76, 124.64, 123.78, 93.79, 51.14, 48.71 and 16.64. Anal. Calc. for: $\left(\mathrm{C}_{27} \mathrm{H}_{23} \mathrm{ClN}_{6} \mathrm{O}\right)$ (M.W. = 482): C, 67.15; H, 4.8; Cl, 7.34; N, 17.4\%; Found: C, 67.42; H, 4.93; N, $17.28 \%$.

3-Phenyl-1-(4-(3-(p-tolyl)-[1,2,4]triazolo[3,4-a]phthalazin-6-yl)piperazin-1-yl)prop2-en-1-one (23): White solid; Yield: $71 \%$; m.p.266-268 ${ }^{\circ} \mathrm{C}$. IR $(\mathrm{KBr}) \mathrm{cm}^{-1}$ : $3020(\mathrm{CH}$ aromatic), 2978 ( $\mathrm{CH}$ aliphatic), $1647(\mathrm{C}=\mathrm{O}), 1604\left(\mathrm{C}=\mathrm{C}\right.$ aromatic). ${ }^{1} \mathrm{H}$ NMR (DMSO$\left.\mathrm{d}_{6}\right) \delta$ ppm: $8.55(\mathrm{~d}, 1 \mathrm{H}, J=7.8), 8.28(\mathrm{~d}, 2 \mathrm{H}, J=7.8), 8.23(\mathrm{~d}, 1 \mathrm{H}, J=8.1), 8.03($ $\mathrm{t}, 1 \mathrm{H}, J=7.4), 7.92$ ( t $, 1 \mathrm{H}, J=7.4), 7.76(\mathrm{~d}, 2 \mathrm{H}, J=6.7), 7.56(\mathrm{~d}, 1 \mathrm{H}, J=15.4), 7.41-$ $7.35(\mathrm{~m}, 6 \mathrm{H}), 4.05(\mathrm{~s}, 2 \mathrm{H}), 3.92(\mathrm{~s}, 2 \mathrm{H}), 3.44(\mathrm{~s}, 4 \mathrm{H}), 2.4(\mathrm{~s}, 3 \mathrm{H}) . \mathrm{MS}(\mathrm{m} / \mathrm{z}): 474$ $\left(\mathrm{C}_{29} \mathrm{H}_{26} \mathrm{~N}_{6} \mathrm{O}, 100 \%, \mathrm{M}^{+}\right) 329\left(\mathrm{C}_{20} \mathrm{H}_{19} \mathrm{~N}_{5}, 17.15 \%\right), 260\left(\mathrm{C}_{16} \mathrm{H}_{12} \mathrm{~N}_{4}, 26.66 \%\right), 113$ $\left(\mathrm{C}_{5} \mathrm{H}_{9} \mathrm{~N}_{2} \mathrm{O}, 32.29 \%\right) .{ }^{13} \mathrm{C}$ NMR (DMSO-d 6$) \delta$ ppm: 165.23, 157.67, 148.17, 143.24,142.35, 140.13, 135.51, 134.03, 131.23, 130.13, 129.82, 129.27, 128.53, 127.64, 127.25, 124.63, 124.09, 123.54, 119.85, 118.51, 51.56, 45.17, 41.83 and 21.48. Anal. Calc. for: $\left(\mathrm{C}_{29} \mathrm{H}_{26} \mathrm{~N}_{6} \mathrm{O}\right)(\mathrm{M} . W .=474)$ : C, 73.4; H, 5.52; N, 17.71\%; Found: C, 73.28; H, $5.68 ; \mathrm{N}, 17.89 \%$.

3-(p-Tolyl)-6-(4-tosylpiperazin-1-yl)-[1,2,4]triazolo[3,4-a]phthalazine (24): White

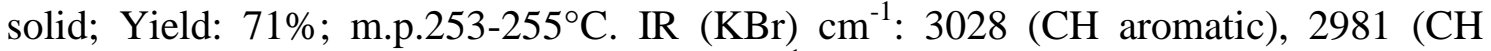
aliphatic), $1674(\mathrm{C}=\mathrm{O}), 1589\left(\mathrm{C}=\mathrm{C}\right.$ aromatic). ${ }^{1} \mathrm{H}$ NMR (DMSO-d $\left.{ }_{6}\right) \delta$ ppm: $8.50(\mathrm{~d}, 1 \mathrm{H}$, $J=7.9), 8.24$ (d, 2H, $J=7.9), 8.06$ (d, 2H, $J=8.1), 7.98$ (t, 1H, $J=7.9), 7.82$ (t, $1 \mathrm{H}, J=$ $8.0 \mathrm{H}), 7.72(\mathrm{~d}, 1 \mathrm{H}, J=7.9), 7.51(\mathrm{~d}, 2 \mathrm{H}, J=7.8), 7.42(\mathrm{~d}, 2 \mathrm{H}, J=7.8), 3.47(\mathrm{~s}, 4 \mathrm{H})$, $3.24(\mathrm{~s}, 4 \mathrm{H}), 2.43(\mathrm{~s}, 3 \mathrm{H})$. MS (m/z): $498\left(\mathrm{C}_{27} \mathrm{H}_{26} \mathrm{~N}_{6} \mathrm{O}_{2} \mathrm{~S}, 13.71 \%, \mathrm{M}^{+}\right) 407$ $\left(\mathrm{C}_{20} \mathrm{H}_{19} \mathrm{~N}_{6} \mathrm{O}_{2} \mathrm{~S}, 57.81 \%\right), 381\left(\mathrm{C}_{19} \mathrm{H}_{19} \mathrm{~N}_{5} \mathrm{O}_{2} \mathrm{~S}, 100 \%\right), 343\left(\mathrm{C}_{20} \mathrm{H}_{19} \mathrm{~N}_{6}, 29.51 \%\right), 254$ $\left(\mathrm{C}_{13} \mathrm{H}_{14} \mathrm{~N}_{6}, 26.04 \%\right), 123\left(\mathrm{C}_{5} \mathrm{H}_{7} \mathrm{~N}_{4}, 12.66 \%\right) .{ }^{13} \mathrm{C}$ NMR (DMSO-d 6 ) $\delta$ ppm: 130.46, 
129.84, 128.14, 127.61, 50.58, 45.9 and 21.49. Anal. Calc. for: $\left(\mathrm{C}_{27} \mathrm{H}_{26} \mathrm{~N}_{6} \mathrm{O}_{2} \mathrm{~S}\right)(\mathrm{M} . \mathrm{W} .=$ 498): C, 65.04; H, 5.26; N, 16.86\%; Found: C, 65.31; H, 5.39; N, 16.81; S, 6.57\%.

Anticancer activity: All the synthesized compounds were subjected to MTT proliferation assay to investigate their in-vitro cytotoxic activity. Hepatocellular carcinoma (HePG-2), mammary gland breast cancer (MCF-7), Human prostate cancer (PC3) and colorectal carcinoma (HCT-116) cell lines was chosen for investigation.

\section{Conclusion}

a series of triazolophthalazine incorporating piperazine derivatives was synthesized and evaluated for their anticancer activity against four human cancer cell lines (Hepatocellular carcinoma (HePG-2), mammary gland breast cancer (MCF-7), Human prostate cancer (PC3) and Colorectal carcinoma (HCT-116)). The results of anticancer evaluation showed that most of the synthesized compounds displayed moderate cytotoxic activities against the selected cell lines. Compound $\mathbf{2 3}$ showed the highest inhibitory effect followed by compound $\mathbf{2 4}$ against hepatocellular carcinoma and colorectal carcinoma cell lines.

\section{REFERENCES}

Abd alla, Mosaad S. M., Mohamed I. Hegab, Nageh A. Abo Taleb, Sherifa M. Hasabelnaby, and A. Goudah. 2010. "Synthesis and Anti-Inflammatory Evaluation of Some Condensed [4-(3,4-Dimethylphenyl)-1(2H)-oxo-phthalazin2-Y1]Acetic Acid Hydrazide." European Journal of Medicinal Chemistry 45(4):1267-77.

Abou-Seri, Sahar M., Wagdy M. Eldehna, Mamdouh M. Ali, and Dalal A. Abou El Ella. 2016. "1-Piperazinylphthalazines as Potential VEGFR-2 Inhibitors and Anticancer Agents: Synthesis and in Vitro Biological Evaluation.” European Journal of Medicinal Chemistry 107:165-79.

Almahli, Hadia, Elie Hadchity, Maiy Y. Jaballah, Racha Daher, Hazem A. Ghabbour, Maha M. Kabil, Nasser S. Al-shakliah, and Wagdy M. Eldehna. 2018. "Development of Novel Synthesized Phthalazinone-Based PARP-1 Inhibitors with Apoptosis Inducing Mechanism in Lung Cancer." Bioorganic Chemistry 77:443-56.

Asif, M. 2012. "Some Recent Approaches of Biologically Active Substituted Pyridazine and Phthalazine Drugs." Current Medicinal Chemistry 19(18):2984-91.

Awadallah, Fadi M., Wafaa I. El-Eraky, and Dalia O. Saleh. 2012. "Synthesis, Vasorelaxant Activity, and Molecular Modeling Study of Some New Phthalazine Derivatives.” European Journal of Medicinal Chemistry 52:14-21.

Boraei, Ahmed T. A., Pankaj K. Singh, Mario Sechi, and Sandro Satta. 2019. "Discovery of Novel Functionalized 1,2,4-Triazoles as PARP-1 Inhibitors in Breast Cancer: Design, Synthesis and Antitumor Activity Evaluation.” European 
Journal of Medicinal Chemistry 182:111621.

Chetan, Bhadaliya, Mahesh Bunha, Monika Jagrat, Barij Nayan Sinha, Philipp Saiko, Geraldine Graser, Thomas Szekeres, Ganapathy Raman, Praveen Rajendran, Dhatchana Moorthy, Arijit Basu, and Venkatesan Jayaprakash. 2010. "Design, Synthesis and Anticancer Activity of Piperazine Hydroxamates and Their Histone Deacetylase (HDAC) Inhibitory Activity." Bioorganic \& Medicinal Chemistry Letters 20(13):3906-10.

El-Helby, Abdel-Ghany A., Rezk R. A. Ayyad, Helmy Sakr, Khaled El-Adl, Mamdouh M. Ali, and Fathalla Khedr. 2017. "Design, Synthesis, Molecular Docking, and Anticancer Activity of Phthalazine Derivatives as VEGFR-2 Inhibitors." Archiv Der Pharmazie 350(12):1700240.

El-Helby, Abdel-Ghany A., Helmy Sakr, Rezk R. A. Ayyad, Khaled El-Adl, Mamdouh M. Ali, and Fathalla Khedr. 2018. "Design, Synthesis, In Vitro Anti-Cancer Activity, ADMET Profile and Molecular Docking of Novel Triazolo[3,4-a]Phthalazine Derivatives Targeting VEGFR-2 Enzyme." AntiCancer Agents in Medicinal Chemistry 18(8):1184-96.

Fedorov, Oleg, Hannah Lingard, Chris Wells, Octovia P. Monteiro, Sarah Picaud, Tracy Keates, Clarence Yapp, Martin Philpott, Sarah J. Martin, Ildiko Felletar, Brian D. Marsden, Panagis Filippakopoulos, Susanne Müller, Stefan Knapp, and Paul E. Brennan. 2014. "[1,2,4]Triazolo[4,3a]Phthalazines: Inhibitors of Diverse Bromodomains." Journal of Medicinal Chemistry 57(2):462-76.

Gurdal, Enise, Ebru Buclulgan, Irem Durmaz, Rengul Cetin-Atalay, and Mine Yarim. 2015. "Synthesis and Anticancer Activity Evaluation of Some Benzothiazole-Piperazine Derivatives." Anti-Cancer Agents in Medicinal Chemistry 15(3):382-89.

Haack, Thomas, Raimondo Fattori, Mauro Napoletano, Franco Pellacini, Giovanni Fronza, Giuseppina Raffaini, and Fabio Ganazzoli. 2005. "Phthalazine PDE IV Inhibitors: Conformational Study of Some 6-Methoxy-1,4-Disubstituted Derivatives.” Bioorganic \& Medicinal Chemistry 13(14):4425-33.

Holló, Berta, József Magyari, Vukosava Živković-Radovanović, Gordana Vučković, Zoran D. Tomić, Imre Miklós Szilágyi, György Pokol, and Katalin Mészáros Szécsényi. 2014. "Synthesis, Characterisation and Antimicrobial Activity of Bis(Phthalazine-1-Hydrazone)-2,6-Diacetylpyridine and Its Complexes with CoIII, NiII, CuII and ZnII." Polyhedron 80:142-50.

Khalil, A. M., M. A. Berghot, and M. A. Gouda. 2009. "Synthesis and Antibacterial Activity of Some New Heterocycles Incorporating Phthalazine." European Journal of Medicinal Chemistry 44(11):4448-54.

Liu, Da-Chuan, Guo-Hua Gong, Cheng-Xi Wei, Xue-Jun Jin, and Zhe-Shan Quan. 
2016. "Synthesis and Anti-Inflammatory Activity Evaluation of a Novel Series of 6-Phenoxy-[1,2,4]Triazolo[3,4-a]Phthalazine-3-Carboxamide Derivatives." Bioorganic \& Medicinal Chemistry Letters 26(6):1576-79.

Lu, Wenchao, Rukang Zhang, Hao Jiang, Huimin Zhang, and Cheng Luo. 2018. "Computer-Aided Drug Design in Epigenetics." Frontiers in Chemistry 6.

Mosmann, T. 1983. "Rapid Colorimetric Assay for Cellular Growth and Survival: Application to Proliferation and Cytotoxicity Assays." Journal of Immunological Methods 65(1-2):55-63.

Moustakim, Moses, Peter G. K. Clark, Laura Trulli, Angel L. Fuentes de Arriba, Matthias T. Ehebauer, Apirat Chaikuad, Emma J. Murphy, Jacqui Mendez-Johnson, Danette Daniels, Chun-Feng D. Hou, Yu-Hui Lin, John R. Walker, Raymond Hui, Hongbing Yang, Lucy Dorrell, Catherine $M$. Rogers, Octovia P. Monteiro, Oleg Fedorov, Kilian V. M. Huber, Stefan Knapp, Jag Heer, Darren J. Dixon, and Paul E. Brennan. 2017. "Discovery of a PCAF Bromodomain Chemical Probe." Angewandte Chemie 129(3):84549.

Moustakim, Moses, Peter G.K. Clark, Laura Trulli, Angel L. Fuentes de Arriba, Matthias T. Ehebauer, Apirat Chaikuad, Emma J. Murphy, Jacqui Mendez-Johnson, Danette Daniels, Chun Feng D. Hou, Yu Hui Lin, John R. Walker, Raymond Hui, Hongbing Yang, Lucy Dorrell, Catherine M. Rogers, Octovia P. Monteiro, Oleg Fedorov, Kilian V. M. Huber, Stefan Knapp, Jag Heer, Darren J. Dixon, and Paul E. Brennan. 2017. "Discovery of a PCAF Bromodomain Chemical Probe.” Angewandte Chemie - International Edition 56(3):827-31.

Olmo, Esther del, Bianca Barboza, Ma Inés Ybarra, José Luis López-Pérez, Rosalía Carrón, Ma Angeles Sevilla, Cinthia Boselli, and Arturo San Feliciano. 2006. "Vasorelaxant Activity of Phthalazinones and Related Compounds.” Bioorganic \& Medicinal Chemistry Letters 16(10):2786-90.

Salvi, Vijay K., Dinesh Bhambi, Jawahar L. Jat, and Ganpat L. Talesara. 2006. "Synthesis and Antimicrobial Activity of Some 2- [ 1- ( 4-Oxo-3 , 4- and Their Imidoxy Derivatives.” 2006(xiv):133-40.

Sun, Xian-Yu, Chuan Hu, Xian-Qing Deng, Cheng-Xi Wei, Zhi-Gang Sun, and Zhe-Shan Quan. 2010. "Synthesis and Anti-Inflammatory Activity Evaluation of Some Novel 6-Alkoxy(Phenoxy)-[1,2,4]Triazolo[3,4-a]Phthalazine-3-Amine Derivatives.” European Journal of Medicinal Chemistry 45(11):4807-12.

Sun, Xian-Yu, Cheng-Xi Wei, Xian-Qing Deng, Zhi-Gang Sun, and Zhe-Shan Quan. 2010. "Evaluation of the Anticonvulsant Activity of 6-(4Chlorophenyoxy)-Tetrazolo[5,1-a]Phthalazine in Various Experimental Seizure Models in Mice." Pharmacological Reports 62(2):273-77. 
Sun, Xian-Yu, Cheng-Xi Wei, Xian-Qing Deng, Zhi-Gang Sun, and Zhe-Shan Quan. 2011. "Synthesis and Primary Anticonvulsant Activity Evaluation of 6Alkyoxyl-Tetrazolo[5,1-a]Phthalazine Derivatives." Arzneimittelforschung 60(06):289-92.

Tarzia, Giorgio, Emilio Occelli, Emilio Toja, Domenico Barone, Nerina Corsico, Licia Gallico, and Franco Luzzani. 1988. "6-(Alkylamino)-3-Aryl-1,2,4Triazolo[3,4- $a]$ Phthalazines. A New Class of Benzodiazepine Receptor Ligands." Journal of Medicinal Chemistry 31(6):1115-23.

Xue, Deng-Qi, Xu-Yao Zhang, Chao-Jie Wang, Li-Ying Ma, Nan Zhu, Peng He, Kun-Peng Shao, Peng-Ju Chen, Yi-Fei Gu, Xiao-Song Zhang, Cai-Feng Wang, Cong-Hui Ji, Qiu-Rong Zhang, and Hong-Min Liu. 2014. "Synthesis and Anticancer Activities of Novel 1,2,4-Triazolo[3,4-a]Phthalazine Derivatives.” European Journal of Medicinal Chemistry 85:235-44.

مشتقات التريازولوفثالازين المتصلة ببيبرازين: التشييد و دراسة النشاط المضاد للسرطان

$$
\begin{aligned}
& \text { 'عبد الله السيد تركى، 'أثنرف حسن بيومى، 'عادل حمدى غياتى، ','حمادة السيد أبو الخير } \\
& \text { 'قسم الكيمياء العضوية الصيدلية، كلية الصيدلة، جامعة الأزهر ـ القاهرة ـ مصر } \\
& \text { كَّم الكيمياء الصيدلية، كلية الصيدلة، جامعة حورس - دمياط الجديد - مصر }
\end{aligned}
$$

hamadaorganic@azhar.edu.eg : البريد الالكتروني للباحث الرئيسي

يُعدُّ السَّرطان أحدُ الأسبابِ الرئيسيَّةُ للوفاةِ في جميع أنحاء العالم خلال العقد الماضي. في محاولة لتطوير عامل

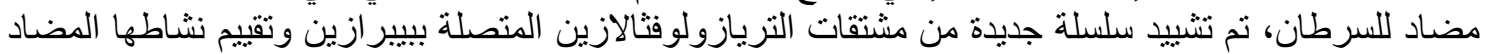

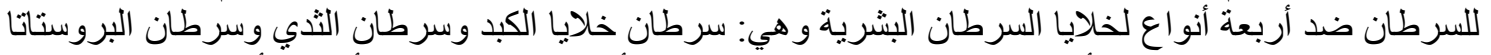

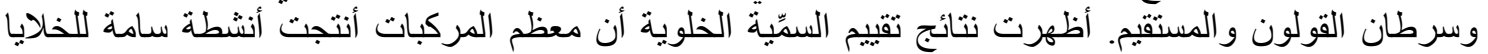

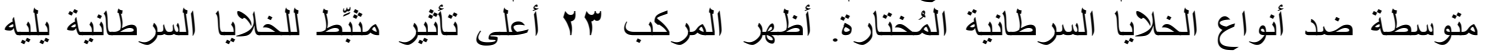

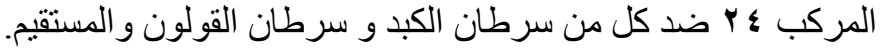

الكلمات المفتاحية: المضاد للسرطان، سرطان خلايا الكبد ،سرطان الثدي ،سرطان البروستاتا ،سرطان القولون 